\title{
HYSTERESIS ASSUMPTIONS FOR PROPELLER IN YAW IN CLOSE STALL CONDITIONS
}

\author{
D. Rozehnal*, V. B. Nguyen**, J. Hnidka****
}

\begin{abstract}
For small unmanned aerial vehicles (UAVs), which typically feature a fixed-pitch propeller, the appropriate selection of the propeller design can lead to increase of thrust and flight efficiency. The current analytical models of propeller display inherent uncertainty, which close to stall conditions is dominated by the dynamic stall phenomenon. The paper presents oscillating airfoil theory and Joukowsky vortex theory, and discusses problems of oscillations close to the stall conditions.
\end{abstract}

Keywords: propeller in yaw, Joukowsky vortex theory, oscillating propeller, Viterna-Corrigan extrapolation, hysteresis.

\section{Introduction}

With the expansion of usage of propeller driven unmanned aerial vehicles (UAVs), the focus of development is shifting to increasing the efficiency of the propulsion. This endeavour is met with difficulties, because of the inaccuracies in current analysis models. Especially fixed-pitch propellers operating in yaw - i.e. forward flight of any multirotor - lead to high angles of attack (AoA) well close to the stall conditions. In or near stall conditions the uncertainty of analytical models is dominated by the fact that rotating airfoils produce larger lift and have larger stall angle than stationary ones. (Lindenberg, 2004).

This phenomenon can be calculated by implementing the Navier-Stokes equations or analytical methods. While CFD is highly efficient in fixed winged application, modelling a rotor wing aircraft is challenging as demonstrated by Kusyumov et. al. (2017). This paper presents analytical Joukowsky vortex method with assumption of low Reynolds numbers $\left(<10^{6}\right)$ and presents some problems with calculating propellers operating in close stall conditions.

\section{Theoretical analysis of the oscillating airfoil}

For a fixed-pitch propeller in yaw the static pre-stall aerodynamic data typically used in aircraft industry can be insufficient, because the local AoA changes with the azimuthal position of the blade $(\Psi)$. Because of this oscillation, an effect called dynamic stall can occur at high AoA (Nguyen, 2017). Both lift coefficient and stall angle are increased for a short period of time - in some cases this increase of the lift coefficient can be up to $100 \%$ (Sharma, 2010).

Nguyen (2017) presents a study in which an airfoil oscillates from $\alpha_{\min }$ to $\alpha_{\max }$ at a constant angular frequency $\omega$. The oscillation of the airfoil can be expressed as

$$
\alpha=\alpha_{0}+\Delta \alpha \sin (\omega t)
$$

\footnotetext{
doc. Ing Dalibor Rozehnal, Ph.D.: Department of Air Force and Aircraft Technology, University of Defence, Kounicova 65 602 00; Brno, CZ, dalibor.rozehnal@unob.cz

** Ing. Van Bang Nguyen: Department of Air Force and Aircraft Technology, University of Defence, Kounicova 65, 602 00; Brno, CZ, vanbang.nguyen@unob.cz

*** Ing. Jakub Hnidka: Department of Air Force and Aircraft Technology, University of Defence, Kounicova 65, 602 00; Brno, CZ, jakub.hnidka@unob.cz
} 
where $\alpha_{0}$ is the mean value of AoA and $\Delta \alpha$ is the amplitude of the movement

$$
\Delta \alpha=\left(\alpha_{\max }-\alpha_{\min }\right) / 2
$$

Typically, rather than angular frequency $\omega$, a reduced frequency $k$ is used

$$
k=\omega c / 2 V
$$

where $c$ is chord length and $V$ is the inflow velocity.

The dynamic stall phenomenon is showed in the Figure 1. For low values of $k$ the deviation of $C_{L}$ from the static values is negligible. However, as the $k$ increases, the oscillating change of the AoA causes hysteresis of the $C_{L}$, which follows a closed loop pattern. This effect is further amplified by the $\alpha_{o}$ and $\Delta \alpha$.
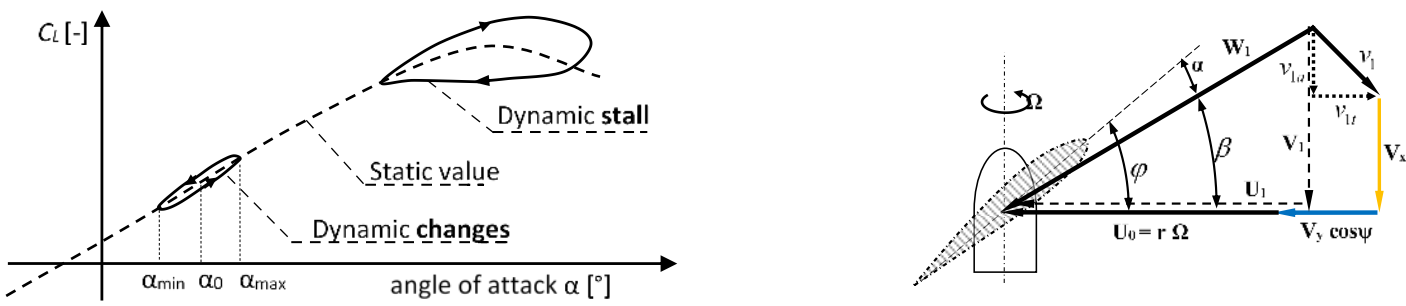

Fig. 1: The dynamic stall phenomenon (left)(Ahlund, 2004) and the Joukowsky vortex theory (right)

\section{Joukowsky propeller characteristic calculation method}

The Joukowsky vortex theory simplifies a rotor wing and replaces it by a system of vortexes with analogues behavior. The velocity induced by the rotor is calculated from the circulation, and subsequently circulation affects the velocity field around the rotor.

During the calculation, the rotor is divided into finite number of sections. In each section are assumed constant geometric (thickness, chord) and aerodynamic characteristics (polar). The local inflow velocity $w_{l}$ is composed from velocities $V_{l}$ and $U_{l}$ with an angle between them equal to the difference between propeller adjustment angle $\varphi$ and local angle of attack (AoA) $\alpha$ as shown in the Figure 1 .

$$
\begin{gathered}
w_{1}=\sqrt{V_{1}^{2}+U_{1}^{2}} \\
\beta=\varphi-\alpha=\arctan \left(V_{1} / U_{1}\right)
\end{gathered}
$$

It can be shown that both $V_{l}$ and $U_{l}$ are dependent on circulation $\Gamma$. Moreover, the circulation $\Gamma$ can be obtained as:

$$
\Gamma=c_{L} c \frac{U_{1}}{\cos (\varphi-\alpha)} \mathrm{D} \Omega
$$

Where $c$ is chord length, $D$ is rotor diameter, $\Omega$ is angular velocity of the rotor and the $C_{L}(\alpha)$ is the lift coefficient.

This algorithm leads to iterative solution of $\Gamma$. This iteration typically converges very quickly. Each calculation of circulation $\Gamma$ from (6) depends on the polar of the given rotor section. Because the rotor geometry changes along the radius and the local Reynolds number changes as well, each section has a unique polar. The polar data for each section can be obtained either by calculation or by wind tunnel experiments. Most of the measurements and calculations are performed in pre-stall conditions. As described by Nguyen (2016) a full polar is a necessity. Therefore and extrapolation is needed.

Extrapolation methods are further discussed by Nguyen (2016) and Mahumuddin et. al. (2017). For purposes of this article a Viterna-Corrigan extrapolation is implemented, as described by Viterna and Corrigan (1982), with flat plane theory expansion for AoA higher than $90^{\circ}$.

The pre-stall data were obtained by calculation performed in XFOIL, while Petinrin and Onoja (2017) performed numerical study and Ostowari and Naik (1985) performed wind tunnel experiments. The last work also studied the post-stall effects up to the AoA of $+110^{\circ}$. The pre-stall data were further extrapolated by Viterna-Corrigan method. 


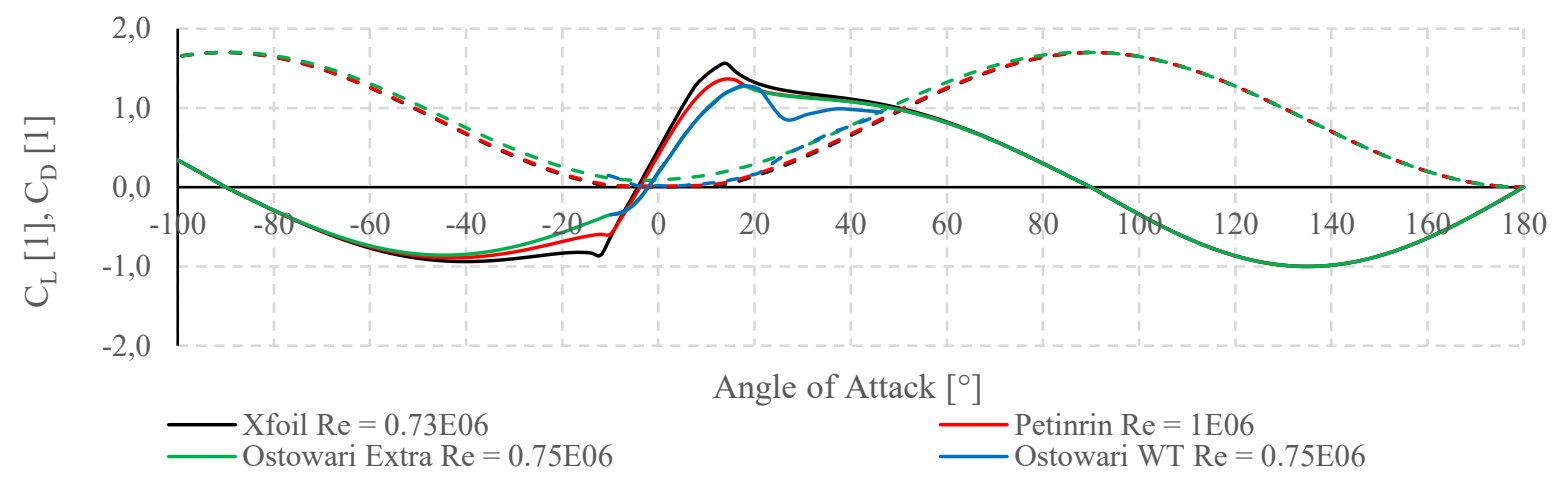

Fig. 2: NACA 4412 airfoil data extended to $\pm 360^{\circ}$ for Reynolds numbers of less than $10^{6}, A R=\infty$ (Solid line $C_{L}$, dashed $C_{D}$ )

The results shown in the Figure 2 correspond well with the results presented by MacNeil and Verstraete (2016) with small deviations caused by lower Reynolds numbers. The numerical solution seems to better correspond with measured results for pre-stall data than XFOIL. XFOIL overpredicts the value of $\mathrm{C}_{L, \max }$ and typically is unable to predict the stall conditions for negative AoA due to the convergence issues. The CFD method underpredicts the AoA at which the $C_{L, \max }$ occurs, but the compliance with the measurement is better.

During the static measurements of the polar above the $C_{L, \max }$ occurs a flow separation, which is coupled with considerable loss of $C_{L}$ and rise of $C_{D}$. The Viterna-Corrigan extrapolation method smoothens out this recovery, causing the largest deviation of calculated and measured curves in the Figure 2. However, the oscillating airfoil follows the static characteristics only under special conditions. Typically, the dynamic stall phenomenon further affects the pre-stall data - the stall AoA and $C_{L}$ both increase and the characteristics closer resemble those predicted by flat plane theory with increased the $\alpha_{\text {stall }}$ (Rozehnal, 2009).

\section{The rotational effect consideration}

The Joukowsky vortex theory had been applied to calculate the local values of AoA for each section of the propeller in yaw. The theory largely relays on the polar data, on which no correction of the rotational effects was applied for this analysis. Moreover, the Joukowsky theory assumes the undisturbed velocity flow in front of each blade - i.e. no interaction between blades.

The local values of AoA $\alpha$ can be used to calculate angular frequency $\omega^{\prime}$, which can be defined as:

$$
\omega^{\prime}=\frac{d \alpha}{d t}=\frac{d \alpha 2 \pi n}{d \psi}
$$

Where $n$ are the revolutions of the propeller. The reduced frequency $k^{\prime}$ can be calculated as

$$
k^{\prime}=\frac{\omega^{\prime} c}{2 V}=\frac{d \alpha \pi n c}{d \psi V}
$$

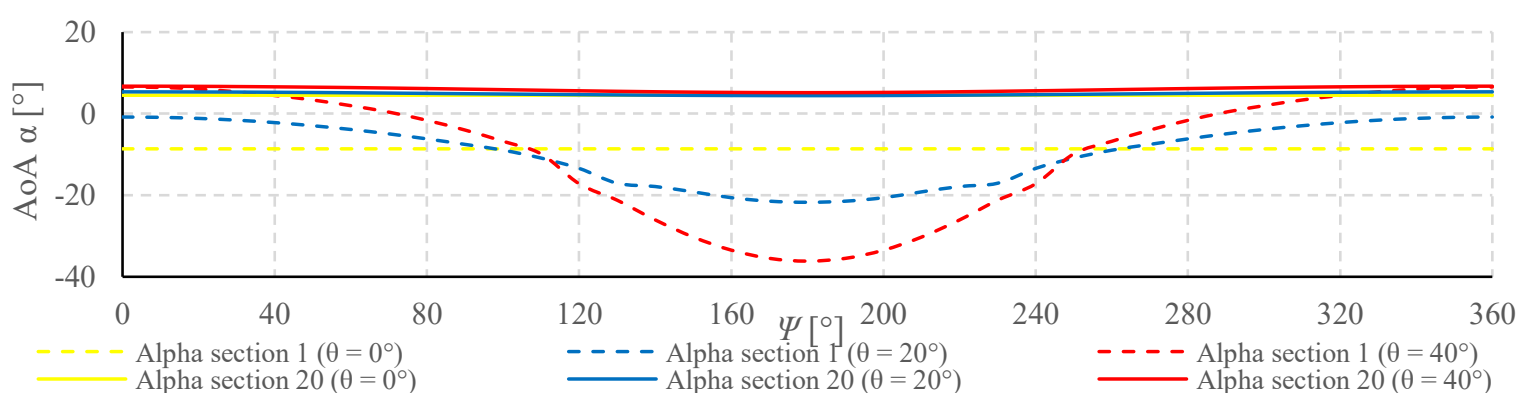

Fig. 3: The change of the local AoA in the first and final section of the blade along the $\Psi(\lambda=0,4)$

The correction of input polar for dynamic stall is affected by the amplitude of the movement $\Delta \alpha$, the mean value of $\alpha_{0}$ and reduced frequency $k^{\prime}$. For uniform inflow parallel to the axis of rotation $\left(\Theta=0^{\circ}\right)$, the value 
of $k^{\prime}$ is constant and no oscillation occurs, as shown in the Figure 4 (tip speed ratio $\lambda=0.4$ ). Figure 3 shows the change of local AoA in the first and final (20) section of the rotor along the azimuthal angle $\Psi$. Increasing the inflow angle $\Theta$ causes the propeller to oscillate (yaw conditions). For relatively small values of $\Theta$ the local AoA does not surpasses the $\alpha_{\text {stall }}$ and the propeller sections follow the oscillating airfoil theory. However, for larger $\Theta$ the stall condition disturb the oscillation and a non-harmonic change of the reduced frequency can be observed (Figure 4). This phenomenon can be observed closer to the hub of the propeller, because the rake tend to be larger at the hub than at the tip. As the pitch is set to larger values, the close stall condition can occur even further on the blade.

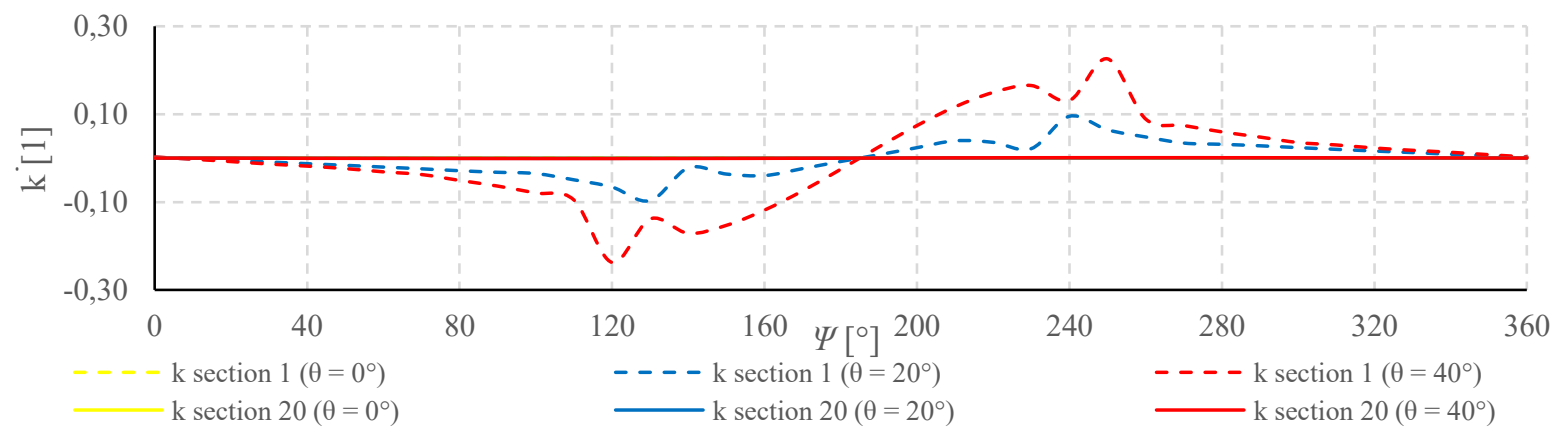

Fig. 4: The change of the $k$ in the first and final section of the blade along the $\Psi(\lambda=0,4)$

\section{Conclusion}

This paper highlights the dependency of analytical models (Joukowsky, or BEM) on airfoil polar. Typically, this polar is only static, which leads to non-standard behavior, as demonstrated in Figure 3 and Figure 4. The correction of airfoil coefficients can be performed by oscillating airfoil theory - however, the theory typically assumes harmonic change of the AoA (and its derivation) - this assumption does not seem to be correct for propellers operating close to stall.

\section{Acknowledgement}

This research was conducted in cooperation with company ENERGOKLASTR.

\section{References}

Åhlund, K. (2004) Investigation of the NREL NASA/Ames Wind Turbine Aerodynamics Database. Aeronautics, FFA, Swedish Defense Research Agency, 2004.

Bang, N. V. and Rozehnal, D. (2016) Determine $360^{\circ}$ polar for calculation of wind turbines. 17th Int. Conf. Transfer 2016, Trenčín, pp.1-7.

Bang, N. V. and Rozehnal, D. (2017) Aerodynamic hysteresis of oscillating airfoil. In: Engineering Mechanics 2017, Brno University of Technology, Brno, pp.106-109.

Kusyumov, A., Kusyumov, S., Mikhailov, S., Romanova, E., Phayzullin, K, Lopatin, E. and Barakos, G. (2017) Main rotor-body action for virtual blades model. Experimental Fluid Mechanics 2017, Liberec, pp. 334-339

Lindenberg, C. (2004) Modelling of rotational augmentation based on engineering considerations and measurements. European Wind Energy Conference, London

MacNeill R. and Verstraete, D. (2016) Blade element momentum theory extended to model low Reynolds number propeller performance. 20th Australasian Fluid Mechanics Conference.

Mahumuddin, F., Klara, S., Sitepu, H. and Hariyanto, S. (2016) Airfoil lift and drag extrapolation with Viterna and Montgomerie methods. 8th Int. Conf. on Applied Energy, Beijing, pp. 811-816.

Ostowari C., Naik D. (1985) Post-stall wind tunnel data for NACA 44xx series airfoil sections, Solar Energy Institute (SERI).

Petinrin, M. O. and Onoja, V. A. (2017) Computational study of aerodynamic flow over NACA 4412 airfoil. British Journal of Applied Science and Technology, pp 1-11.

Rozehnal, D. (2009) Development of experimental aerodynamics at the University of Defence in Brno. Habilitation thesis, Brno

Sharma, M. (2010) Experimental investigations of dynamic stall for an oscillating airfoil. $\mathrm{PhD}$. Thesis. Indian Institute of Technology, Kanpur.

Viterna, L. A. and Corrigan, R. D. (1982) Fixed pitch rotor performance of large horizontal axis wind turbines. Large Horizontal-Axis Wind Turbines. NASA Conference Publication 2230, Cleveland, pp. 69-85. 